\title{
The period function for second-order quadratic ODEs is monotone*
}

\author{
Armengol Gasull ${ }^{(1)}$, Antoni Guillamon ${ }^{(2)}$ and Jordi Villadelprat ${ }^{(3)}$ \\ (1) Dept. de Matemàtiques, Universitat Autònoma de Barcelona, \\ 08193 Bellaterra, Barcelona, Spain. \\ (2) Dept. de Matemàtica Aplicada I, Universitat Politècnica de Catalunya, \\ Dr. Marañón 44-50, 08028 Barcelona, Spain. \\ (3) Dept. d'Enginyeria Informàtica i Matemàtiques, Universitat Rovira i Virgili, \\ Av. dels Països Catalans 26, 43007 Tarragona, Spain.
}

Dedicated to Professor Jorge Sotomayor on the occasion of his 60th birthday.

\begin{abstract}
Very little is known about the period function for large families of centers. In one of the pioneering works on this problem, Chicone [?] conjectured that all the centers encountered in the family of second-order differential equations $\ddot{x}=V(x, \dot{x})$, being $V$ a quadratic polynomial, should have a monotone period function. Chicone solved some of the cases but some others remain still unsolved. In this paper we fill up these gaps by using a new technique based on the existence of Lie symmetries and presented in [?]. This technique can be used as well to reprove all the cases that were already solved, providing in this way a compact proof for all the quadratic second-order differential equations. We also prove that this property on the period function is no longer true when $V$ is a polynomial which nonlinear part is homogeneous of degree $n>2$.
\end{abstract}

MSC: Primary: 37C-27; Secondary: 34C-25, 34C-14, 34A-26.

\section{Introduction}

Let $p_{0} \in \mathbb{R}^{2}$ be a center of a planar system of differential equations. The period annulus of $p_{0}$, that we denote by $\mathcal{P}$, is defined as the greatest punctured neighborhood of $p_{0}$ foliated by periodic orbits. We take a parameterization of the set of periodic orbits in $\mathcal{P}$, say $s \mapsto \gamma_{s}$, and we consider the period function, $s \mapsto T(s)$, that assigns to each $s$ the period of the periodic orbit $\gamma_{s}$.

*Partially supported by the DGES grant number BFM2002-04236 and CONACIT grant number 2001SGR-00173. 
Several problems around the period function in planar vector fields have been studied in the last half-century, starting from the works of Urabe ([?]), Loud ([?]) and Pleshkan ([?]) on isochronicity (constant period function) in specific families of planar vector fields. Later on, the problem of the monotonicity of the period function attracted the attention of Coppel and Gavrilov (for potential systems, see [?]) and Waldwogel (for the Lotka-Volterra systems, see [?]), to quote significant examples, among others. Maybe, the most interesting contributions come from Chicone, see [?], [?], [?](with Jacobs), who studied not only the monotonicity but also computed the so-called period constants, and used them to study bifurcations of the period function in parametric families as well as boundary value problems. Coming back to the problem of isochronicity, in the early nineties, Villarini ([?]) and Sabatini ([?]) related the problem of isochronicity to the existence of Lie symmetries (see [?] for a survey on isochronicity) and, more recently, these ideas have been applied to the study of the monotonicity ([?]).

In this paper we study the period function of the centers of quadratic systems that come from second-order ODEs $\ddot{x}=V(x, \dot{x})$, that is,

$$
\left\{\begin{array}{l}
\dot{x}=y, \\
\dot{y}=-x+a x^{2}+b x y+c y^{2},
\end{array}\right.
$$

with $a^{2}+b^{2}+c^{2} \neq 0$.

It is well known (see [?] for instance) that any center in this family can be brought, by means of a coordinate transformation into one of the following two forms:

$$
\begin{aligned}
& \left\{\begin{array}{l}
\dot{x}=y, \\
\dot{y}=-x+a x^{2}+b x y-a y^{2} ;
\end{array}\right. \\
& \left\{\begin{array}{l}
\dot{x}=y, \\
\dot{y}=-x+a x^{2}+c y^{2},
\end{array}\right.
\end{aligned}
$$

where $a, b$ and $c$ are arbitrary real numbers.

For many subcases, which will be revisited in Section ??, Chicone gave a proof of the monotonicity of the period function (see [?, ?]). As far as we know there are, though, some that were not proved yet:

Case I System (??) with $a \neq 0$,

Case II System (??) with $a c<0$.

As we will see, the system of Case I can be easily brought to a system which is already known to have a center with monotonic period function. Thus, essentially, just one case remains unsolved. By means of a new technique for showing the monotonicity of the period function, we solve this last case and we give shorter proofs for the others. We can state therefore: 
Theorem 1.1. If the origin is a center for system (??), then the associated period function is increasing.

Chicone [?] has conjectured that if a quadratic system has a center with a period function which is not monotonic then, by an affine transformation and a constant rescaling of time, it can be brought to the Loud normal form

$$
\left\{\begin{array}{l}
\dot{x}=-y+B x y \\
\dot{y}=x+D x^{2}+F y^{2}
\end{array}\right.
$$

and that the period function of these centers has at most two critical periods. In view of Theorem ??, with a rescaling, this conjecture is reduced to the case $B=1$. On the other hand, it is well known (see [?]) that the centers of $\ddot{x}=-x+\tilde{V}(x, \dot{x})$, with $\tilde{V}$ being a cubic polynomial without constant and linear terms, may have a non monotonic period function. In addition, we prove the following result:

Proposition 1.2. For any $m \geq 3$, there are (reversible) centers of the form

$$
\left\{\begin{array}{l}
\dot{x}=y \\
\dot{y}=-x+V_{m}(x, y)
\end{array}\right.
$$

where $V_{m}$ is a homogeneous polynomial of degree $m$, with a non monotonic period function.

The paper is organized in the following way. In Section ?? we present the techniques used to prove Theorem ?? and we give new proofs of the cases already solved. Sections ?? and ?? are devoted respectively to show the monotonicity in Case I and Case II. Finally, in Section ?? we prove Proposition ??.

\section{Previous results}

The mentioned techniques to ensure the monotonicity of the period function are based on the following result, which is proved in [?].

Theorem 2.1. Let $p$ be a center of a $\mathcal{C}^{1}$ vector field $X$ and let $\mathcal{P}$ denote its period annulus. Let $U$ be $\mathcal{C}^{1}$ vector field on $\mathcal{P} \cup\{p\}$, transversal to $X$ on $\mathcal{P}$, and such that $[X, U]=\mu X$ on $\mathcal{P}$ for some $\mathcal{C}^{1}$ function $\mu$ on $\mathcal{P} \cup\{p\}$. Then, if $\psi(s)$ is a trajectory of $U$, for any $s_{0}$ such that $\psi\left(s_{0}\right) \in \mathcal{P}$ it holds

$$
T^{\prime}\left(s_{0}\right)=\int_{0}^{T\left(s_{0}\right)} \mu\left(x\left(t ; s_{0}\right), y\left(t ; s_{0}\right)\right) d t
$$

where $\left(x\left(t ; s_{0}\right), y\left(t ; s_{0}\right)\right)$ is the periodic orbit of $X$ such that $\left(x\left(0 ; s_{0}\right), y\left(0 ; s_{0}\right)\right)=\psi\left(s_{0}\right)$ and $T\left(s_{0}\right)$ is its period. 
For Theorem ?? to be useful we need to be able to compute $\mu$ and control its integral. It is already known the existence of pairs $(U, \mu)$ satisfying $[X, U]=\mu X$ for sufficiently regular vector fields $X$ with a non-degenerate center. From a geometrical point of view, the vector field $U$ is the infinitesimal generator of the Lie group of symmetries of $X$. We will also take advantage of the next remark.

Remark 2.2 Let $U$ be a vector field transversal to $X$ such that $[X, U]=\mu X$ for some $\mathcal{C}^{1}$ function $\mu$. If we consider $U^{\star}=U+g X$, where $g$ is any $\mathcal{C}^{1}$ function, then $U^{\star}$ is also transversal to $X$ and $\left[X, U^{\star}\right]=\mu^{\star} X$ with $\mu^{\star}=\mu+(\nabla g)^{t} \cdot X$.

The usefulness of Remark ?? lies in the fact that once we now a pair $(\mu, U)$ for a given vector field $X$, we can generate other pairs by adding "multiples" of $X$ to $U$ and modifying $\mu$ concordantly.

In the rest of this section, for the sake of completeness and to show the efficiency of the method just presented, we apply it to reprove some cases already solved by Chicone.

\subsection{System (??)}

In order to prove the monotonicity of the period function of the center of (??) we consider the following four cases:

$$
\begin{aligned}
& a=0 \text { and } c \neq 0 \\
& a \neq 0 \text { and } c=0 \\
& a c>0 \\
& a c<0
\end{aligned}
$$

Chicone solved the first and second cases in [?], and the third one in [?]. As we already mentioned, in Section ?? we shall solve the fourth case.

In order to reprove the case (3.1), note first that by means of the coordinate transformation $\left\{x_{1}=2 c x, y_{1}=2 c y\right\}$ it is enough to consider only the case $c=1 / 2$. Then, renaming the variables as $\{x, y\}$, an additional change of variables, $\left\{w=\log \left(1+x-y^{2} / 2\right), z=y\right\}$, brings the system into

Now one can verify that the vector field

$$
\mathcal{X}:=\left\{\begin{array}{l}
\dot{z}=1-e^{w}, \\
\dot{w}=z .
\end{array}\right.
$$

$$
\mathcal{U}:=\left\{\begin{array}{l}
\dot{z}=\frac{1}{2} z \\
\dot{w}=1-\frac{w}{e^{w}-1}
\end{array}\right.
$$

satisfies that $[\mathcal{X}, \mathcal{U}]=\mu \mathcal{X}$ with

$$
\mu(z, w):=\frac{1-e^{2 w}+2 w e^{w}}{2\left(e^{2 w}-2 e^{w}+1\right)} .
$$


Proposition 11.a in [?] shows that the integral of $\mu$ along the orbits of $\mathcal{X}$ is always positive and so, from Theorem ??, it follows that the period function is increasing.

In the case (3.2) the change of variables $\left\{x_{1}=-a x, y_{1}=-a y\right\}$ allows to consider only the case $a=-1$. Renaming the variables as $\{x, y\}$, one can show that the new vector field, say $\mathcal{X}$, is transversal to

$$
\mathcal{U}:=\left\{\begin{array}{l}
\dot{x}=\frac{3 x+2 x^{2}}{6(1+x)} \\
\dot{y}=\frac{1}{2} y
\end{array}\right.
$$

and that $[\mathcal{X}, \mathcal{U}]=\mu \mathcal{X}$ with

$$
\mu(x, y)=-\frac{x(2+x)}{6(1+x)^{2}}
$$

Then Proposition 11.b in [?] shows that the integral of $\mu$ along the orbits of $\mathcal{X}$ is always positive and so, again from Theorem ??, the period function is increasing.

The proof of the case (3.3) using Theorem ?? presents the same kind of difficulties than for the case (3.4), which is solved in Section ??, and so, for the sake of shortness, we prefer to avoid it in this paper.

\subsection{System (??)}

In order to prove the monotonicity of the period function of the center of (??) we consider the following two cases:

$$
\begin{aligned}
& a=0 \\
& a \neq 0
\end{aligned}
$$

Chicone proved in [?] the monotonicity of the period function in the case (2.1). In order to reprove it by means of Theorem ?? we proceed as follows. The coordinate transformation $\left\{x_{1}=-b x, y_{1}=\right.$ $-b y\}$ allows to consider only the case $b=-1$. Then, renaming the variables as $\{x, y\}$, another change of variables, $\{z=x, w=\log (1+y), \tau=-t\}$, brings the system into

$$
\mathcal{X}:=\left\{\begin{array}{l}
\dot{z}=1-e^{w} \\
\dot{w}=z
\end{array}\right.
$$

This is the same vector field that we obtained in the case (3.1) and so the result follows. Next section is devoted to prove the case (2.2). 


\section{Proof of the monotonicity in Case I}

By means of the coordinate transformation $\left\{x_{1}=a x, y_{1}=a y\right\}$ system (??) can also be reduced to the case $a=1$ without loss of generality. We therefore consider

$$
\left\{\begin{array}{l}
\dot{x}=y \\
\dot{y}=-x+x^{2}+b x y-y^{2} .
\end{array}\right.
$$

One can verify that the change of variables

$$
\left\{z=-x-\left(b+\sqrt{b^{2}+1}\right) y, w=-x-\left(b-\sqrt{b^{2}+1}\right) y\right\}
$$

brings system (??) to

$$
\left\{\begin{array}{l}
\dot{z}=-w(1+z)\left(\sqrt{b^{2}+1}+b\right) \\
\dot{w}=z(1+w)\left(\sqrt{b^{2}+1}-b\right)
\end{array}\right.
$$

This is a Lotka-Volterra system and it is well-known (see [?] for instance) that the centers of these systems have an increasing period function. For the sake of compactness, let us point out that this fact was also proved in [?] directly from Theorem ??.

\section{Proof of the monotonicity in Case II}

\subsection{Reduction of the problem through Theorem ??}

We first note that, rescaling the variables, we can assume, without loss of generality, that $a=1$ and $c<0$. We consider therefore

$$
\left\{\begin{array}{l}
\dot{x}=y \\
\dot{y}=-x+x^{2}+c y^{2} .
\end{array}\right.
$$

A computation shows that $H(x, y)=A(x)+C(x) y^{2}$, with

$$
A(x):=\frac{e^{-2 c x}}{4 c^{2}}\left(2 c x^{2}+2(1-c) x-1+1 / c\right)+\frac{c-1}{4 c^{3}} \text { and } C(x):=\frac{1}{2} e^{-2 c x},
$$

is a first integral of (??), and one can verify that $\kappa(x):=-e^{-2 c x}$ is its corresponding integrating factor (see [?] for details). We shall take advantage of this fact to perform a coordinate transformation that brings (??) to a potential system. This follows from the next result.

Lemma 4.1. Suppose that a given planar differential system has a first integral of the form $H(x, y)=A(x)+B(x) y+C(x) y^{2}$ and that its corresponding integrating factor, say $\kappa$, depends only on $x$. Then, if $\kappa(x) C(x) \neq 0$, the coordinate transformation given by

$$
u=f(x):=\int_{0}^{x} \frac{\kappa(s)}{\sqrt{2 C(s)}} d s \text { and } v=\frac{2 C(x) y+B(x)}{\sqrt{2 C(x)}}
$$


brings the system to

$$
\left\{\begin{array}{l}
\dot{u}=-v \\
\dot{v}=g\left(f^{-1}(u)\right)
\end{array}\right.
$$

where

$$
g(x):=\frac{B(x)\left(B(x) C^{\prime}(x)-2 C(x) B^{\prime}(x)\right)}{2 \kappa(x) \sqrt{2 C(x)^{3}}}+\frac{\sqrt{2 C(x)} A^{\prime}(x)}{\kappa(x)} .
$$

Proof. Note first of all that we can write the given differential system as

$$
\left\{\begin{array}{l}
\dot{x}=\frac{-H_{y}(x, y)}{\kappa(x)}, \\
\dot{y}=\frac{H_{x}(x, y)}{\kappa(x)},
\end{array}\right.
$$

since, by hypothesis, $H$ is a first integral and $\kappa$ is its corresponding integrating factor. It is clear moreover that we can assume without loss of generality that $C(x)>0$. In this case we can rewrite the first integral as

$$
H(x, y)=\frac{1}{2}\left(\sqrt{2 C(x)} y+\frac{B(x)}{\sqrt{2 C(x)}}\right)^{2}+\frac{4 A(x) C(x)-B(x)^{2}}{4 C(x)} .
$$

In order to obtain the desired coordinate transformation, we define

$$
v:=\sqrt{2 C(x)} y+\frac{B(x)}{\sqrt{2 C(x)}}
$$

and then find $u(x, y)$ such that

$$
\left|\begin{array}{ll}
u_{x} & u_{y} \\
v_{x} & v_{y}
\end{array}\right|=\kappa(x) \text { for all } x \text {. }
$$

The simplest way to achieve this is by choosing $u(x, y)=f(x)$ so that $f^{\prime}(x) \sqrt{2 C(x)}=\kappa(x)$, which yields to

$$
u=f(x):=\int_{0}^{x} \frac{\kappa(s)}{\sqrt{2 C(x)}} d s
$$

Finally, some computations show that this coordinate transformation brings (??) to the potential system given in the statement.

By applying Lemma ?? to system (??) it turns out that the coordinate transformation

$$
\left\{u=\frac{e^{-c x}-1}{c}, v=y e^{-c x}\right\}
$$


brings it to the potential system

$$
\left\{\begin{array}{l}
\dot{u}=-v \\
\dot{v}=(1+c u) \ln (1+c u)(c+\ln (1+c u)) / c^{2}
\end{array}\right.
$$

that is, a Hamiltonian system with Hamiltonian function $H(u, v)=\frac{1}{2} v^{2}+F(u)$, where

$$
\begin{aligned}
F(u):=\frac{1}{4 c^{3}} & \left(2 \ln ^{2}(1+c u)+4 u c \ln ^{2}(1+c u)+2 u^{2} c^{2} \ln ^{2}(1+c u)-2 \ln (1+c u)\right. \\
& -4 u c \ln (1+c u)-2 u^{2} c^{2} \ln (1+c u)+2 c u+u^{2} c^{2}+2 c \ln (1+c u) \\
& \left.+4 u c^{2} \ln (1+c u)+2 u^{2} c^{3} \ln (1+c u)-2 u c^{2}-u^{2} c^{3}\right) .
\end{aligned}
$$

The above change of coordinates already appears in [?]. It is easy to check that if $X$ is the potential vector field associated to $H(u, v)=v^{2}+F(u)$, then $[X, U]=\mu X$, where $U$ is the vector field associated to the system $\dot{u}=F(u) / F^{\prime}(u), \dot{v}=v / 2$ and

$$
\mu(u)=\left(\frac{F(u)}{F^{\prime}(u)}\right)^{\prime}-1 / 2 .
$$

In our case a computation shows that

$$
\mu(u)=-\frac{c+2}{6} u+\mathrm{O}\left(u^{2}\right) .
$$

Thus, since we look for some $\mu>0$, we shall take advantage of Remark ?? to remove the linear term in $\mu$. The choice $g(u, v)=(c+2) v / 6$ (in the notation of Remark ??) provides

$$
\mu^{\star}(u):=\mu(u)+\frac{c+2}{6} F^{\prime}(u)=\frac{2 c^{2}+5 c+5}{6} u^{2}+\mathrm{O}\left(u^{3}\right) .
$$

Note in particular that $2 c^{2}+5 c+5>0$ for any $c$.

Let us denote by $\left(u_{L}, u_{R}\right)$ the projection of the period annulus of the center at the origin of system (??) onto the $u$-axis. It is clear, on account of Theorem ??, that to show the monotonicity of its period function it is enough to verify that $\mu^{\star}(u)>0$ for all $u \in\left(u_{L}, u_{R}\right) \backslash\{0\}$. To do so it is first necessary to study the ranges of $\left(u_{L}, u_{R}\right)$ for the different values of $c$. It is easy to show that

$$
\begin{aligned}
& u_{L}=\frac{e^{-c}-1}{c} \text { and } u_{R}<-\frac{1}{c} \text { in case that } c \in(-1,0), \\
& u_{L}=\frac{1}{c}\left(e^{\frac{1-c-\sqrt{c^{2}-1}}{2}}-1\right) \text { and } u_{R}=-\frac{1}{c} \text { in case that } c \leq-1 .
\end{aligned}
$$

Now, in order to simplify the formulae that we shall obtain, we perform the change of variable $u=\left(e^{-x}-1\right) / c$, which one can verify that drives to

$$
\mu_{2}(x):=\mu^{\star}(u(x))=\frac{e^{2 x}}{12 c^{2} x^{2}(c-x)^{2}} \sum_{i=0}^{6} C_{i}(x) x^{i},
$$


with

$$
\begin{array}{llrl}
C_{0}(x) & =3 c^{3}(c-1)\left(e^{-2 x}-1\right), & & C_{1}(x)=3 c^{2}(c-1)\left(c+c e^{-2 x}-2 e^{-2 x}+2\right), \\
C_{2}(x) & =3 c^{2}(1-c)\left(1+3 e^{-2 x}\right), & C_{3}(x) & =2 c^{2}\left(3 e^{-2 x}-c^{2} e^{-3 x}-2 c e^{-3 x}\right), \\
C_{4}(x) & =6 c^{2} e^{-3 x}(c+2), & & C_{5}(x)=-6 c e^{-3 x}(c+2), \\
C_{6}(x) & =2 e^{-3 x}(c+2) . & &
\end{array}
$$

Consequently, taking (??) into account, we must prove that

$$
\begin{aligned}
& \mu_{2}(x)>0 \text { for all } x \in(c,+\infty) \backslash\{0\} \text { in case that } c \in(-1,0), \\
& \mu_{2}(x)>0 \text { for all } x \in\left(\frac{c-1+\sqrt{c^{2}-1}}{2},+\infty\right) \backslash\{0\} \text { in case that } c \leq-1 .
\end{aligned}
$$

The following two subsections are devoted to study these cases.

\subsection{The case $c \in(-1,0)$}

We study the cases $x \in(c, 0)$ and $x \in(0,+\infty)$ separately.

\subsubsection{The study of $\mu_{2}$ on $x \in(0,+\infty)$}

We shall prove the following result.

Proposition 4.2. If $c \in(-1,0)$ then $\mu_{2}(x)>0$ for all $x>0$.

Note that, for the values of $c$ under consideration, $C_{5}(x)>0$ and $C_{6}(x)>0$ for all $x>0$. Hence, on account of the expression of $\mu_{2}$ given in (??), Proposition ?? will follow if we show that

$$
T(x):=C_{0}(x)+C_{1}(x) x+C_{2}(x) x^{2}+C_{3}(x) x^{3}+C_{4}(x) x^{4}
$$

is positive on $(0,+\infty)$. To do so we proceed as follows. One can verify that

$$
T(x)=c^{2}\left(D_{0}(x)+D_{1}(x) c+D_{2}(x) c^{2}\right),
$$

where

$$
\begin{aligned}
& D_{0}(x)=-6 x+3 x^{2}+\left(6 x+9 x^{2}+6 x^{3}\right) e^{-2 x}+12 x^{4} e^{-3 x}, \\
& D_{1}(x)=3+3 x-3 x^{2}-\left(3+9 x+9 x^{2}\right) e^{-2 x}+\left(-4 x^{3}+6 x^{4}\right) e^{-3 x}, \\
& D_{2}(x)=-3+3 x+(3+3 x) e^{-2 x}-2 x^{3} e^{-3 x} .
\end{aligned}
$$


Consequently, since

$$
D_{0}(x)+D_{1}(x) c+D_{2}(x) c^{2}=\left(4 c^{2}+10 c+10\right) x^{4}+\mathrm{O}\left(x^{5}\right)
$$

and $\left(4 c^{2}+10 c+10\right)>0$ for $c \in(-1,0)$, it is clear that Proposition ?? will follow once we prove the following two lemmas.

Lemma 4.3. $D_{1}(x)^{2}-4 D_{0}(x) D_{2}(x)<0$ for all $x \in(0,2)$.

Lemma 4.4. $D_{0}(x)>0, D_{1}(x)<0$ and $D_{2}(x)>0$ for all $x \geq 2$.

Proof of Lemma ?? We will prove in fact that $S(x):=\left(D_{1}(x)^{2}-4 D_{0}(x) D_{2}(x)\right) e^{6 x}$ is negative. To this end note first that we can write

$$
S(x)=P_{0}(x)+P_{1}(x) e^{x}+P_{2}(x) e^{2 x}+P_{3}(x) e^{3 x}+P_{4}(x) e^{4 x}+P_{6}(x) e^{6 x}
$$

with

$$
\begin{array}{ll}
P_{0}(x)=4 x^{6}(3 x+2)^{2}, & P_{1}(x)=-12 x^{3}\left(5 x^{3}+9 x^{2}+5 x-2\right), \\
P_{2}(x)=9 x^{4}-18 x^{3}-45 x^{2}-18 x+9, & P_{3}(x)=-12 x^{3}\left(3 x^{3}+5 x^{2}-9 x+2\right), \\
P_{4}(x)=-18\left(x^{2}+x-1\right)\left(x^{2}+3 x-1\right), & P_{6}(x)=9\left(x^{2}-3 x+1\right)^{2} .
\end{array}
$$

The following table gathers the study of the signum of each polynomial $P_{i}(x)$ on $(0,2)$.

\begin{tabular}{l|c|c|c|c|c|} 
& $P_{1}(x)$ & $P_{2}(x)$ & $P_{3}(x)$ & $P_{4}(x)$ & $P_{6}(x)$ \\
\hline$\left(0, \eta_{1}\right)$ & + & + & - & - & + \\
\hline$\left(\eta_{1}, \eta_{3}\right)$ & - & + & - & - & + \\
\hline$\left(\eta_{3}, \eta_{2}\right)$ & - & + & + & - & + \\
\hline$\left(\eta_{2}, \eta_{6}\right)$ & - & - & + & - & + \\
\hline$\left(\eta_{6}, \eta_{5}\right)$ & - & - & + & + & + \\
\hline$\left(\eta_{5}, \eta_{4}\right)$ & - & - & + & - & + \\
\hline$\left(\eta_{4}, 2\right)$ & - & - & - & - & + \\
\hline
\end{tabular}

In the above table, $\eta_{1}$ and $\eta_{2}$ are respectively the roots of $P_{1}(x)$ and $P_{2}(x)$ in $(0,2)$. The ones of $P_{3}(x)$ are $\eta_{3}$ and $\eta_{4}$. Finally, $\eta_{5}$ and $\eta_{6}$ are the ones of $P_{4}(x)$. Let us note that these roots can be obtained algebraically. Their approximate values are:

$$
\begin{array}{lll}
\eta_{1} \approx 0.260, & \eta_{2} \approx 0.282, & \eta_{3} \approx 0.269, \\
\eta_{4} \approx 0.880, & \eta_{5} \approx 0.618, & \eta_{6} \approx 0.303 .
\end{array}
$$

During the proof we will also use that the polynomials

$$
m(x)=1+x+\frac{1}{2} x^{2}+\frac{1}{3 !} x^{3}+\frac{1}{4 !} x^{4}+\frac{1}{5 !} x^{5}+\frac{1}{6 !} x^{6}+\frac{1}{7 !} x^{7}+\frac{1}{8 !} x^{8}
$$


and

$$
M(x)=1+x+\frac{1}{2} x^{2}+\frac{1}{3 !} x^{3}+\frac{1}{4 !} x^{4}+\frac{1}{5 !} x^{5}+\frac{1}{6 !} x^{6}+\frac{1}{7 !} x^{7}+\frac{8}{8 !} x^{8}
$$

verify that $0<m(x)<e^{x}<M(x)$ for all $x \in(0,2)$. These polynomial bounds, obtained by means of Taylor's expansion, are the ones of lowest degree which are efficient for our purpose.

On account of the above table and using the polynomial bounds of $e^{x}$, it follows that the polynomial

$$
B_{1}(x)=P_{0}(x)+P_{1}(x) M(x)+P_{2}(x) M(x)^{2}+P_{3}(x) m(x)^{3}+P_{4}(x) m(x)^{4}+P_{6}(x) M(x)^{6}
$$

is a upper bound of $S(x)$ on $\left(0, \eta_{1}\right)$. This is a polynomial of degree 52 that can be written as

$$
B_{1}(x)=x^{8}\left(B_{11}(x)+B_{12}(x)\right),
$$

where the coefficients of all the monomials of $B_{11}$ (respectively $B_{12}$ ) are negative (respectively positive). Therefore

$$
S(x)<B_{1}(x)<x^{8}\left(B_{11}(0)+B_{12}\left(\eta_{1}\right)\right)<x^{8}\left(B_{11}(0)+B_{12}(3 / 10)\right)<x^{8}(-59+1)=-58 x^{8}<0
$$

for all $x \in\left(0, \eta_{1}\right)$. An alternative way to show this fact would be of course to use Sturm's algorithm. Exactly the same procedure shows that $S(x)<0$ in all the other intervals and it is not included here for the sake of shortness.

Proof of Lemma ?? The fact that $D_{0}(x)>0$ for all $x \geq 2$ is clear. To prove that $D_{1}(x)$ is negative let us first note that $D_{1}(x)=10 x^{4}+\mathrm{O}\left(x^{5}\right)$. On the other hand, by means of Bolzano's Theorem, we can assert that $D_{1}(x)$ has at least one root on $(1,3 / 2)$. So it is clear that it suffices to prove that $D_{1}(x)$ has at most five zeros (counting multiplicities) on the half straight line $x \geq 0$. This fact will follow once we check that the function

$$
\frac{d^{5}}{d x^{5}}\left(D_{1}(x) e^{2 x}\right)=e^{-x}\left(Q_{1}(x)+Q_{2}(x) e^{3 x}\right),
$$

where $Q_{1}(x):=-6 x^{4}+124 x^{3}-780 x^{2}+1680 x-960$ and $Q_{2}(x):=-96 x^{2}-384 x-144$, does not vanish for $x \geq 0$. Indeed, since $Q_{2}(x)<0$ for $x \geq 0$, and $Q_{1}(x)+Q_{2}(x)$ is a fourth degree polynomial with no real roots and negative for all $x \in \mathbb{R}$, it turns out that

$$
\frac{d^{5}}{d x^{5}}\left(D_{1}(x) e^{2 x}\right)<e^{-x}\left(Q_{1}(x)+Q_{2}(x)\right)<0 \text { for all } x \geq 0 .
$$

We must show finally that $D_{2}(x)>0$ for all $x>0$. Again, one can verify that $D_{2}(x)=$ $4 x^{4}+\mathrm{O}\left(x^{5}\right)$ and, on the other hand,

$$
\frac{d^{4}}{d x^{4}}\left(D_{2}(x) e^{3 x}\right)=(15+3 x) e^{x}+(81+243 x) e^{3 x}>0 \text { for all } x \geq 0 .
$$

This concludes the proof of the result. 


\subsubsection{The study of $\mu_{2}$ on $x \in(c, 0)$}

Let $\widetilde{\mu}_{2}$ denote the function obtained after the change of variables $(x, c) \longmapsto(-x,-c)$ into the expression of $\mu_{2}$ given in (??). Thus, for each $c \in(0,1)$, we have to prove that $\widetilde{\mu}_{2}(x)>0$ for all $x \in(0, c)$. A computation shows that we can write

$$
\widetilde{\mu}_{2}(x)=\frac{1}{12 c^{2} x^{2}(c-x)^{2}}\left(P_{0}(x)+P_{1}(x) e^{x}+P_{2}(x) e^{-2 x}\right),
$$

where

$$
\begin{aligned}
& P_{0}(x)=3 c^{2}\left(c+c^{2}-\left(3 c+2+c^{2}\right) x+3(c+1) x^{2}-2 x^{3}\right), \\
& P_{1}(x)=2 x^{3}(2-c)(x-c)^{3} \\
& P_{2}(x)=3 c^{2}(c+1)\left(-c+(2-c) x+x^{2}\right) .
\end{aligned}
$$

We prove the following result:

Lemma 4.5. For each $c \in(0,1), P_{0}(x)+P_{1}(x) e^{x}+P_{2}(x) e^{-2 x}>0$ for all $x \in(0, c)$.

Proof. Note first that $P_{1}$ is negative on the region under consideration and that $P_{2}$ may be positive or negative. By means of the Taylor's expansion one can easily show that

$$
e^{x}<M_{1}(x) \text { and } m_{2}(x)<e^{-2 x}<M_{2}(x) \text { for all } x \in(0,1),
$$

where

$$
\begin{aligned}
& M_{1}(x)=1+x+\frac{1}{2} x^{2}+\frac{1}{6} x^{3}+\frac{1}{8} x^{4}, \\
& M_{2}(x)=1-2 x+2 x^{2}-\frac{4}{3} x^{3}+\frac{2}{3} x^{4}, \\
& m_{2}(x)=1-2 x+2 x^{2}-\frac{4}{3} x^{3}+\frac{2}{3} x^{4}-\frac{4}{15} x^{5} .
\end{aligned}
$$

Moreover, on account of $(0, c) \subset(0,1)$, it follows that the polynomials

$$
B_{1}(x)=P_{0}(x)+P_{1}(x) M_{1}(x)+P_{2}(x) m_{2}(x)
$$

and

$$
B_{2}(x)=P_{0}(x)+P_{1}(x) M_{1}(x)+P_{2}(x) M_{2}(x)
$$

are respectively lower bounds of $P_{0}(x)+P_{1}(x) e^{x}+P_{2}(x) e^{-2 x}$ on the regions where $P_{2}$ is positive and negative. We shall prove that, for each $c \in(0,1)$, these two polynomials are positive for all $x \in(0, c)$. To this end we first perform the change of variable $x=c y$. So we have to show that

$$
V_{1}(y):=B_{1}(c y) \text { and } V_{2}(y):=B_{2}(c y)
$$


are positive for all $y \in(0,1)$. Finally we make a change on the parameter by introducing $b$ satisfying $b=\psi(c):=c /(1-c)$. Then one can check that $V_{1}$ and $V_{2}$ become respectively

$$
\widetilde{V}_{1}(y)=\frac{b^{6} y^{4}}{60(b+1)^{11}}\left(R_{0}(y)+R_{1}(y) b+R_{2}(y) b^{2}+R_{3}(y) b^{3}+R_{4}(y) b^{4}+R_{5}(y) b^{5}\right)
$$

and

$$
\widetilde{V}_{2}(y)=\frac{b^{6} y^{4}}{12(b+1)^{11}}\left(S_{0}(y)+S_{1}(y) b+S_{2}(y) b^{2}+S_{3}(y) b^{3}+S_{4}(y) b^{4}+S_{5}(y) b^{5}\right),
$$

where

$$
\begin{aligned}
& R_{0}(y)=600-720 y+240 y^{2}, \\
& R_{1}(y)=2400-2520 y+360 y^{2}+240 y^{3}, \\
& R_{2}(y)=3840-3432 y-216 y^{2}+480 y^{3}+120 y^{4}, \\
& R_{3}(y)=3120-2388 y-556 y^{2}+252 y^{3}+180 y^{4}+40 y^{5}, \\
& R_{4}(y)=1320-960 y-156 y^{2}-114 y^{3}+150 y^{4}-30 y^{5}+30 y^{6}, \\
& R_{5}(y)=240-204 y+64 y^{2}-111 y^{3}+45 y^{4}-25 y^{5}+15 y^{6},
\end{aligned}
$$

and

$$
\begin{aligned}
& S_{0}(y)=120-144 y+48 y^{2}, \\
& S_{1}(y)=480-504 y+72 y^{2}+48 y^{3}, \\
& S_{2}(y)=768-696 y-24 y^{2}+96 y^{3}+24 y^{4}, \\
& S_{3}(y)=624-516 y-44 y^{2}+60 y^{3}+36 y^{4}+8 y^{5}, \\
& S_{4}(y)=264-240 y+36 y^{2}+6 y^{3}+30 y^{4}-6 y^{5}+6 y^{6}, \\
& S_{5}(y)=48-60 y+32 y^{2}-3 y^{3}+9 y^{4}-5 y^{5}+3 y^{6} .
\end{aligned}
$$

Since $\psi((0,1))=(0,+\infty)$, the problem is now to prove that, for each $b>0$, the polynomials $\widetilde{V}_{1}(y)$ and $\widetilde{V}_{2}(y)$ are positive for all $y \in(0,1)$. To this end it is enough to show that, for all $y \in(0,1)$, $R_{i}(y)>0$ and $S_{i}(y)>0$, and this can be done for instance by means of Sturm's algorithm.

\subsection{The case $c<-1$}

Recall that in this case we have to prove that $\mu_{2}(x)>0$ for all $x \in\left(\frac{c-1+\sqrt{c^{2}-1}}{2},+\infty\right) \backslash\{0\}$. Hence, since

$$
\frac{c-1+\sqrt{c^{2}-1}}{2} \in(-1,-1 / 2) \text { for all } c<-1 \text {, }
$$


it suffices to show that $\mu_{2}(x)>0$ for all $x \in(-1,+\infty) \backslash\{0\}$. To this end we first perform a change in the parameter by introducing $b$ satisfying $b=\varphi(c):=-1 /(c+1)$. Then, $\mu_{2}$ writes as

$$
\mu_{2}(x)=\frac{e^{2 x}}{12(b+1)^{2} x^{2}(b+1+b x)^{2}} \sum_{i=0}^{4} D_{i}(x) b^{i},
$$

where

$$
\begin{aligned}
& D_{0}(x)=-2 x^{3} e^{-3 x}+(3+3 x) e^{-2 x}-3+3 x, \\
& D_{1}(x)=-2 x^{3}(2+3 x) e^{-3 x}+\left(15+21 x+9 x^{2}\right) e^{-2 x}-15+9 x+3 x^{2}, \\
& D_{2}(x)=-6 x^{4}(x+1) e^{-3 x}+\left(27+51 x+36 x^{2}+6 x^{3}\right) e^{-2 x}-27+3 x+12 x^{2}, \\
& D_{3}(x)=-2 x^{3}(x-2)(x+1)^{2} e^{-3 x}+\left(21+51 x+45 x^{2}+12 x^{3}\right) e^{-2 x}-21-9 x+15 x^{2}, \\
& D_{4}(x)=2 x^{3}(x+1)^{3} e^{-3 x}+6(x+1)^{3} e^{-2 x}-6-6 x+6 x^{2} .
\end{aligned}
$$

Consequently, since $\varphi((-\infty,-1))=(0,+\infty)$, we aim to prove that, for each $b>0$, we have $\mu_{2}(x)>0$ for all $x \in(-1,+\infty) \backslash\{0\}$. This is done in the following two sections.

\subsubsection{The study of $\mu_{2}$ on $x \in(0,+\infty)$.}

Lemma 4.6. $D_{i}(x)>0$ for all $x>0$.

Proof. That $D_{0}(x)>0$ for all $x>0$ follows from using that $D_{0}(x)=4 x^{4}+\mathrm{O}\left(x^{5}\right)$ and

$$
\frac{d^{4}}{d x^{4}}\left(D_{0}(x) e^{3 x}\right)=(81+243 x) e^{3 x}+(15+3 x) e^{x}>0 \text { for all } x \geq 0 .
$$

The same method works for $D_{1}$ because $D_{1}(x)=6 x^{4}+\mathrm{O}\left(x^{5}\right)$ and, on the other hand,

$$
\frac{d^{4}}{d x^{4}}\left(D_{1}(x) e^{3 x}\right)=\left(81+1377 x+243 x^{2}\right) e^{3 x}+\left(207+93 x+9 x^{2}\right) e^{x}-144>0 \text { for } x \geq 0 .
$$

Let us turn next to study $D_{2}$. In this case $D_{2}(x)=4 x^{4}+\mathrm{O}\left(x^{5}\right)$ and, defining $P_{0}(x):=-144-$ $144 x+1008 x^{2}-624 x^{3}+114 x^{4}-6 x^{5}$ and $P_{1}(x):=240+816 x+192 x^{2}$, we have that

$$
\frac{d^{4}}{d x^{4}}\left(D_{2}(x) e^{2 x}\right)=e^{-x}\left(P_{0}(x)+P_{1}(x) e^{3 x}\right) .
$$

Then, since for all $x \geq 0$ we have that $P_{1}(x)>0$ and $e^{3 x} \geq 1+3 x+9 / 2 x^{2}+9 / 2 x^{3}$, we can assert that

$$
\begin{aligned}
P_{0}(x)+P_{1}(x) e^{3 x} & \geq P_{0}(x)+P_{1}(x)\left(1+3 x+9 / 2 x^{2}+9 / 2 x^{3}\right) \\
& =858 x^{5}+4650 x^{4}+4704 x^{3}+4728 x^{2}+1392 x+96,
\end{aligned}
$$


which is clearly positive for all $x \geq 0$. The same method shows that $D_{3}(x)>0$ for all $x>0$. Indeed, $D_{3}(x)=6 x^{4}+\mathrm{O}\left(x^{5}\right)$ and, on the other hand,

$$
\frac{d^{4}}{d x^{4}}\left(D_{3}(x) e^{2 x}\right)=e^{-x}\left(P_{2}(x)+P_{3}(x) e^{3 x}\right)
$$

where $P_{2}(x):=48-432 x-336 x^{2}+868 x^{3}-354 x^{4}+48 x^{5}-2 x^{6}$ and $P_{3}(x):=96+816 x+240 x^{2}$. Again, for all $x \geq 0, P_{3}(x)>0$ and since $e^{3 x} \geq 1+3 x+9 / 2 x^{2}+9 / 2 x^{3}+27 / 8 x^{4}$, it turns out that

$$
\begin{aligned}
P_{2}(x)+P_{3}(x) e^{3 x} & \geq P_{2}(x)+P_{3}(x)\left(1+3 x+9 / 2 x^{2}+9 / 2 x^{3}+27 / 8 x^{4}\right) \\
& =808 x^{6}+3882 x^{5}+4722 x^{4}+5692 x^{3}+2784 x^{2}+672 x+144
\end{aligned}
$$

which is again positive for all $x \geq 0$.

The study of $D_{4}$ is more delicate. Let us note first that we can write it as $D_{4}(x)=P_{4}(x) e^{-3 x}+$ $P_{5}(x) e^{-2 x}+P_{6}(x)$, where

$$
P_{4}(x)=2 x^{3}(x+1)^{3}, P_{5}(x)=6(x+1)^{3} \text { and } P_{6}(x)=-6-6 x+6 x^{2} .
$$

It is clear that $P_{4}$ and $P_{5}$ are positive for all $x>0$. On the other hand, $P_{6}$ is a second degree polynomial which is negative on $(0, \xi)$ and positive on $(\xi,+\infty)$, where $\xi:=\frac{\sqrt{5}+1}{2} \approx 1.618$.

Consequently, for $x \geq \xi$ we can assert that

$$
\begin{aligned}
D_{4}(x) & =\left(P_{4}(x)+P_{5}(x) e^{x}+P_{6}(x) e^{3 x}\right) e^{-3 x}>\left(P_{4}(x)+P_{5}(x)+P_{6}(x)\right) e^{-3 x} \\
& =\left(2 x^{6}+6 x^{5}+6 x^{4}+8 x^{3}+24 x^{2}+12 x\right) e^{-3 x} .
\end{aligned}
$$

This clearly shows that $D_{4}$ is positive for all $x \geq \xi$. In order to prove it on $(0, \xi)$ we shall use that, for all $x \in(0,2)$, it holds $e^{-x}>m(x)>0$ with

$$
m(x)=1-x+\frac{1}{2} x^{2}-\frac{1}{3 !} x^{3}+\frac{1}{4 !} x^{4}-\frac{1}{5 !} x^{5} .
$$

Consequently, since $0<\xi<2$, we can assert that, for all $x \in(0, \xi)$,

$$
\begin{aligned}
D_{4}(x)> & P_{4}(x) m(x)^{3}+P_{5}(x) m(x)^{2}+P_{6}(x) \\
= & -\frac{1}{864000} x^{21}+\frac{1}{72000} x^{20}-\frac{31}{288000} x^{19}+\frac{17}{27000} x^{18}-\frac{169}{57600} x^{17} \\
& +\frac{161}{14400} x^{16}-\frac{2033}{57600} x^{15}+\frac{661}{7200} x^{14}-\frac{911}{4800} x^{13}+\frac{6217}{21600} x^{12}-\frac{547}{2400} x^{11} \\
& -\frac{317}{1200} x^{10}+\frac{629}{480} x^{9}-\frac{1067}{480} x^{8}+\frac{41}{30} x^{7}+\frac{103}{60} x^{6}-\frac{23}{5} x^{5}+4 x^{4} .
\end{aligned}
$$

Now, by means of Sturm's algorithm, one can check that this polynomial is positive on $(0, \xi)$. This concludes the proof of the result. 


\subsubsection{The study of $\mu_{2}$ on $x \in(-1,0)$.}

Since one can check that $D_{0}(x)+D_{1}(x) b+D_{2}(x) b^{2}=\left(4+6 b+4 b^{2}\right) x^{4}+\mathrm{O}\left(x^{5}\right)$, it is clear that, on account of the decomposition of $\mu_{2}$ given in (??), the result will follow once we prove these two lemmas.

Lemma 4.7. $D_{3}(x)>0$ and $D_{4}(x)>0$ for all $x \in(-1,0)$.

Lemma 4.8. $D_{1}(x)^{2}-4 D_{0}(x) D_{2}(x)<0$ for all $x \in(-1,0)$.

Proof of Lemma ?? We shall prove in fact that $\widetilde{D}_{3}(x):=D_{3}(-x)$ and $\widetilde{D}_{4}(x):=D_{4}(-x)$ are positive on $(0,1)$. To this end we will use that, for all $x \in(0,1), 0<m(x)<e^{x}<M(x)$ with

$$
m(x)=1+x+\frac{1}{2} x^{2}+\frac{1}{6} x^{3} \text { and } M(x)=1+x+\frac{1}{2} x^{2}+\frac{1}{2} x^{3} .
$$

Consider first $\widetilde{D}_{3}$, which can be written as

$$
\widetilde{D}_{3}(x)=P_{1}(x) e^{2 x}+P_{2}(x) e^{3 x}+P_{3}(x)
$$

where $P_{1}(x):=21-51 x+45 x^{2}-12 x^{3}, P_{2}(x):=-2 x^{3}(x+2)(x-1)^{2}$ and $P_{3}(x):=-21+9 x+15 x^{2}$.

Since one can easily verify that $P_{1}(x)>0$ and $P_{2}(x)<0$ for all $x \in(0,1)$, by using the upper and lower bounds of $e^{x}$ introduced before it follows that

$$
\begin{aligned}
\widetilde{D}_{3}(x)> & P_{1}(x) m(x)^{2}+P_{2}(x) M(x)^{3}+P_{3}(x) \\
= & -\frac{1}{4} x^{15}-\frac{3}{4} x^{14}-\frac{3}{2} x^{13}-3 x^{12}-\frac{9}{4} x^{11}-\frac{3}{4} x^{10} \\
& +\frac{5}{3} x^{9}+\frac{27}{4} x^{8}+\frac{61}{12} x^{7}+\frac{16}{3} x^{6}+\frac{39}{4} x^{5}+\frac{17}{4} x^{4} \\
= & -\frac{x^{4}}{12}\left(\left(9 x^{6}-51\right)+\left(27 x^{6}-117\right) x+\left(36 x^{6}-64\right) x^{2}\right. \\
& \left.\quad+\left(18 x^{6}-61\right) x^{3}+\left(9 x^{6}-81\right) x^{4}+\left(3 x^{6}-20\right) x^{5}\right)
\end{aligned}
$$

which is clearly positive on $(0,1)$.

Let us turn now to study $\widetilde{D}_{4}$, which can be written as

$$
\widetilde{D}_{4}(x)=P_{4}(x) e^{2 x}+P_{5}(x) e^{3 x}+P_{6}(x),
$$

where $P_{4}(x):=6(1-x)^{3}, P_{5}(x):=2 x^{3}(x-1)^{3}$ and $P_{6}(x):=6 x^{2}+6 x-6$. Hence, $P_{4}(x)>0$ and $P_{5}(x)<0$ on $(0,1)$. Consequently

$$
\begin{aligned}
\widetilde{D}_{4}(x) & >P_{4}(x) m(x)^{2}+P_{5}(x) M(x)^{3}+P_{6}(x) \\
& =\frac{x^{4}}{12}\left(3 x^{11}+9 x^{9}-9 x^{7}-35 x^{5}-6 x^{4}+6 x^{3}-4 x^{2}+66 x+42\right),
\end{aligned}
$$

and it is easy to check (for instance, from its Sturm sequence) that this polynomial takes only positive values on $(0,1)$. 
Proof of Lemma ?? We will show that $\Delta(x):=D_{1}(-x)^{2}-4 D_{0}(-x) D_{2}(-x)$ is negative on $(0,1)$. Since $\Delta(x)=-28 x^{8}+\mathrm{O}\left(x^{9}\right)$, it is clear that the result follows if we prove that

$$
\frac{d^{8}}{d x^{8}}\left(\Delta(x) e^{-3 x}\right)<0 \text { for all } x \in[0,1) .
$$

To this end let us first note that

$$
\frac{d^{8}}{d x^{8}}\left(\Delta(x) e^{-3 x}\right)=\left(P_{0}(x)+P_{2}(x) e^{2 x}+P_{4}(x) e^{4 x}+P_{5}(x) e^{5 x}+P_{6}(x) e^{6 x}\right) e^{-3 x},
$$

where

$$
\begin{aligned}
P_{0}(x)= & 59049 x^{4}-39366 x^{3}-1869885 x^{2}+4028454 x-1156923, \\
P_{2}(x)= & -18 x^{4}+792 x^{3}-11394 x^{2}+62856 x-109962, \\
P_{4}(x)= & 9 x^{4}+414 x^{3}+5715 x^{2}+28242 x+41157, \\
P_{5}(x)= & 3072 x^{6}+46080 x^{5}+125952 x^{4}-774144 x^{3}-4230144 x^{2}-5999616 x-2322432, \\
P_{6}(x)= & -78732 x^{8}-1679616 x^{7}-13611888 x^{6}-53187840 x^{5}, \\
& -104509440 x^{4}-95800320 x^{3}-28304640 x^{2}+5806080 x+2419200 .
\end{aligned}
$$

It can be shown moreover that $P_{2}$ and $P_{5}$ are negative on $[0,1)$ and that $P_{4}$ is positive. On the other hand, for $x \in(0,1)$, we have that

$$
m_{2}(x)<e^{2 x}, m_{5}(x)<e^{5 x}, e^{4 x}<M_{4}(x) \text { and } m_{6}(x)<e^{6 x}<M_{6}(x),
$$

where

$$
\begin{aligned}
& m_{2}(x)=1+2 x+2 x^{2}+4 / 3 x^{3}+2 / 3 x^{4}+4 / 15 x^{5}+4 / 45 x^{6} \\
& m_{5}(x)=1+5 x+25 / 2 x^{2}+125 / 6 x^{3}+625 / 24 x^{4}+625 / 24 x^{5}+3125 / 144 x^{6} \\
& m_{6}(x)=1+6 x+18 x^{2}+36 x^{3}+54 x^{4}+324 / 5 x^{5}+324 / 5 x^{6} \\
& M_{4}(x)=1+4 x+8 x^{2}+32 / 3 x^{3}+32 / 3 x^{4}+128 / 15 x^{5}+2816 / 9 x^{6} \\
& M_{6}(x)=1+6 x+18 x^{2}+36 x^{3}+54 x^{4}+324 / 5 x^{5}+130896 / 5 x^{6} .
\end{aligned}
$$

We obtained these bounds by using the Taylor's expansion of the corresponding functions. Then, for those $x \in[0,1)$ such that $P_{6}(x)>0$, we have that

$$
\begin{aligned}
\frac{d^{8}}{d x^{8}}\left(\Delta(x) e^{-3 x}\right) \leq & \left(P_{0}(x)+P_{2}(x) m_{2}(x)+P_{4}(x) M_{4}(x)+P_{5}(x) m_{5}(x)+P_{6}(x) M_{6}(x)\right) e^{-3 x} \\
= & \left(-\frac{10305703872}{5} x^{14}-\frac{219880525104}{5} x^{13}-\frac{5346920434616}{15} x^{12}\right. \\
& -\frac{6966948024432}{5} x^{11}-\frac{41103195192544}{15} x^{10}-\frac{12590764760928}{5} x^{9} \\
& -\frac{3775600126304}{5} x^{8}+\frac{699698439904}{5} x^{7}+\frac{280999239968}{5} x^{6} \\
& -\frac{16332290304}{5} x^{5}-1090396800 x^{4}-218725632 x^{3} \\
& \left.-14708736 x^{2}+6773760 x-1128960\right) e^{-3 x}
\end{aligned}
$$


and, by applying again Sturm's algorithm, we can assert that this polynomial is negative on $[0,1)$. Finally, for those $x \in[0,1)$ such that $P_{6}(x)<0$ it follows that

$$
\begin{aligned}
\frac{d^{8}}{d x^{8}}\left(\Delta(x) e^{-3 x}\right) \leq & \left(P_{0}(x)+P_{2}(x) m_{2}(x)+P_{4}(x) M_{4}(x)+P_{5}(x) m_{5}(x)+P_{6}(x) m_{6}(x)\right) e^{-3 x} \\
= & \left(-\frac{25509168}{5} x^{14}-\frac{569704752}{5} x^{13}-\frac{14926114808}{15} x^{12}\right. \\
& -\frac{22105379952}{5} x^{11}-\frac{165175393504}{15} x^{10}-\frac{81925377888}{5} x^{9} \\
& -\frac{79806672224}{5} x^{8}-\frac{58413037856}{5} x^{7}-\frac{34880542432}{5} x^{6} \\
& -\frac{16332290304}{5} x^{5}-1090396800 x^{4}-218725632 x^{3} \\
& \left.-14708736 x^{2}+6773760 x-1128960\right) e^{-3 x}
\end{aligned}
$$

and then, again by means of Sturm's algorithm, it can be shown that this polynomial is negative on the interval $[0,1)$. This concludes the proof of the result.

Remark 4.9 In Sections ?? and ?? we considered the cases $c \in(-1,0)$ and $c \in(-\infty,-1)$ respectively. So it remains to study $c=-1$. In this case, from (??), one can check that

$$
\mu_{2}(x)=\frac{e^{2 x}}{12 c^{2} x^{2}(c-x)^{2}}\left(2 x^{3}(x+1)^{3} e^{-3 x}+6(x+1)^{3} e^{-2 x}-6-6 x+6 x^{2}\right) .
$$

This is precisely the function $D_{4}$ introduced at the beginning of Section ??, and the combination of Lemmas ?? and ?? show that it is positive on $(-1,+\infty) \backslash\{0\}$.

The proof of Case II, which clearly contrasts with the simplicity of the statement, is then finished. Of course, the choice of $g$ we have made at the beginning of this section to obtain $\mu_{2}$ is the best we have been able to get, but it does not eliminate the possibility of finding another $g$ that makes $\mu_{2}>0$ more evident. This could provide, then, a shorter and more understandable proof.

\section{$5 \quad$ Proof of Proposition ??}

For any $m \geq 3$, the family of systems with homogeneous nonlinearities

$$
\left\{\begin{array}{l}
\dot{x}=y, \\
\dot{y}=-x+\sum_{i=0}^{[m / 2]} \alpha_{i} x^{m-2 i} y^{2 i},
\end{array}\right.
$$


has a reversible center at the origin. It is worth to mention that in [?] the authors conjecture that the centers of (??) are those systems which are invariant under the changes either $(x, t) \mapsto(-x,-t)$ or $(y, t) \mapsto(-y,-t)$. System (??) corresponds to the second case and, in polar coordinates (after changing the sign of the time for convenience), it writes as

$$
\left\{\begin{array}{l}
\dot{R}=-f(\theta) R^{m} \\
\dot{\theta}=1-g(\theta) R^{m-1}
\end{array}\right.
$$

where $f(\theta)=h(\theta) \sin \theta$ and $g(\theta)=h(\theta) \cos \theta$ with

$$
h(\theta)= \begin{cases}\sum_{k=0}^{m / 2} \beta_{2 k} \cos (2 k \theta), & \text { if } m \text { is even, } \\ \sum_{k=0}^{[m / 2]} \beta_{2 k+1} \cos ((2 k+1) \theta), & \text { if } m \text { is odd. }\end{cases}
$$

The coefficients $\beta_{i} \in \mathbb{R}$ above can be easily obtained from the coefficients $\alpha_{i}$ of the initial system. A classical tool to simplify the study of (??) is to transform it into an Abel equation (see [?]) by means of the change $r=\frac{R^{m-1}}{1-g(\theta) R^{m-1}}$. In our situation one can verify that the Abel equation that we obtain is

$$
\frac{d r}{d \theta}=(1-m) f(\theta) g(\theta) r^{3}+\left(g^{\prime}(\theta)-(m-1) f(\theta)\right) r^{2},
$$

which, in terms of $h$, writes as

$$
\frac{d r}{d \theta}=A(\theta) r^{3}+B(\theta) r^{2},
$$

where $A(\theta):=(1-m) \sin (\theta) \cos (\theta) h^{2}(\theta)$ and $B(\theta):=\cos (\theta) h^{\prime}(\theta)-m \sin (\theta) h(\theta)$. Note that if $r(\theta ; \rho)$ is a solution of this equation with initial condition $r(0 ; \rho)=\rho$, then

$$
r(\theta ; \rho)=\rho+\sum_{k=2}^{\infty} u_{k}(\theta) \rho^{k}
$$

for some functions $u_{k}$ that can be obtained recursively. For instance,

$$
u_{2}(\theta)=\int_{0}^{\theta} B(\psi) d \psi \text { and } u_{3}(\theta)=\int_{0}^{\theta}\left(A(\psi)+2 B(\psi) u_{2}(\psi)\right) d \psi
$$

Now, from the second equation in (??) and using variables $(r, \theta)$ again, we obtain the following expression for the period function of the center at the origin of (??):

$$
T(\rho)=\int_{0}^{2 \pi} \frac{d \theta}{1-g(\theta) R^{m-1}}=\int_{0}^{2 \pi}(1+g(\theta) r) d \theta=2 \pi+\int_{0}^{2 \pi} \cos (\theta) h(\theta)\left\{\rho+\sum_{k \geq 2} u_{k}(\theta) \rho^{k}\right\} d \theta .
$$

We conclude therefore that $T(\rho)=2 \pi+\sum_{i \geq 1} T_{i} \rho^{i}$ with

$$
T_{1}=\int_{0}^{2 \pi} \cos (\theta) h(\theta) d \theta \text { and } T_{k}=\int_{0}^{2 \pi} \cos (\theta) h(\theta) u_{k}(\theta) \rho^{k} d \theta, \text { for } k \geq 2 .
$$


In order to show that there are parameters for which the period function of the center at the origin of (??) is not monotonic, we study the cases $m$ even and $m$ odd separately. In the first case, i.e., $m=2 n$ with $n \geq 2$, we take $h(\theta)=a \cos (2 \theta)+\cos (m \theta)$. Let us assume first that $n \geq 3$. Then, by using the above formulas, some tedious computations show that

$$
\begin{aligned}
& T_{1}=0, \\
& T_{2}=-\frac{1}{3} \frac{\pi\left(2 a^{2} n^{2}-3 a^{2} n-3 n-2 a^{2}\right)}{1+2 n}, \\
& \left.T_{3}\right|_{T_{2}=0}=0, \\
& \left.T_{4}\right|_{T_{2}=0}=\frac{\pi n\left(32 n^{6}-102 n^{5}-59 n^{4}+396 n^{3}-299 n^{2}-132 n+2\right)}{8(n-2)^{2}(n+2)(2 n+1)^{3}} .
\end{aligned}
$$

It can be checked that the polynomial $32 n^{6}-102 n^{5}-59 n^{4}+396 n^{3}-299 n^{2}-132 n+2$ has all its real roots smaller than 3 and, consequently, for all $n \geq 3$, we can assert that $T_{4}>0$ when $T_{2}=0$. To show that there exist critical periods we first observe that $T_{2}$ vanishes at

$$
a_{ \pm}= \pm \sqrt{\frac{3 n}{2 n^{2}-3 n-2}}
$$

and that, on the other hand,

$$
\left.\frac{\partial T_{2}}{\partial a}\right|_{a=a_{ \pm}}=\mp 2 \pi \sqrt{\frac{n(n-2)}{3(2 n-1)}} .
$$

Note that the bifurcation values $a_{ \pm}$and their respective derivatives are well defined for all $n \geq 3$. Thus, taking for instance $a \gtrsim a_{+}$, we will have that $T_{2}<0$ and $T_{4}>0$. Therefore, for this parameter, the corresponding period function has at least one local minimum. Let us study next the case $n=2$ (i.e., $m=4$ ). One can verify that in this case

$$
T_{1}=0, \quad T_{2}=\pi\left(\frac{2}{5}+\frac{a}{2}\right),\left.\quad T_{3}\right|_{T_{2}=0}=0 \text { and }\left.\quad T_{4}\right|_{T_{2}=0}=-\frac{66}{625} \pi .
$$

Taking $a \gtrsim-4 / 5$ we will have that $T_{2}>0$ and $T_{4}<0$ so that the period function has at least one local maximum.

Finally, for an odd $m$, i.e., $m=2 n+1$ with $n \geq 1$, we choose $h(\theta)=a \cos \theta+\cos (m \theta)$. In this case one can verify that

$$
T_{1}=a \pi \text { and }\left.T_{2}\right|_{T_{1}=0}=\pi(2 n+1) 4(n+1)>0 .
$$

It is clear then that, for $a \lesssim 0, T_{1}<0$ and $T_{2}>0$ so that the period function has at least one local minimum. This concludes the proof of Proposition ??.

\section{References}

[1] J. Chavarriga, M. Sabatini A survey of isochronous centers, Qual. Theory Dyn. Syst. 1 (1999), 1-70. 
[2] L.A. Cherkas. Number of limit cycles of an autonomous second-order system. Differ. Eq. 5 (1976), 666-668.

[3] C. Chicone. The monotonicity of the period function for planar Hamiltonian vector fields, J. Differential Equations 69 (1987), 310-321.

[4] C. Chicone. Geometric methods for two-point nonlinear boundary value problems, J. Differential Equations 72 (1988), 360-407.

[5] C. Chicone. Review in MathSciNet, ref. 94h:58072.

[6] C. Chicone, M. Jacobs. Bifurcations of critical periods for plane vector fields, Trans. Amer. Math. Soc. 312 (1989), 433-486.

[7] W.A. Coppel, L. Gavrilov. The period function of a Hamiltonian quadratic system, Differential Integral Equations 6 (1993), 1357-1365.

[8] E. Freire, A. Gasull, A. Guillamon. First derivative of the period function with applications, Preprint (2001).

[9] W.S. Loud. Behavior of the period of solutions of certain plane autonomous systems near centers, Contributions to Differential Equations 3 (1964), 21-36.

[10] V. Lunkevich, K. Sibirskii. Integrals of a general quadratic differential system in cases of a center, Differential Equations 18 (1982), 563-568.

[11] I.I. Pleshkan. A new method of investigating the isochronicity of a system of two differential equations, J. Differential Equations, 5 (1969) 796-802.

[12] C. Rousseau, B. Toni. Local bifurcations of critical periods in the reduced Kukles system, Canad. J. Math. 49 (1997), 338-358.

[13] M. Sabatini. Characterizing isochronous centres by Lie brackets, Diff. Eq. Dyn. Sys., 5, 91-99 (1997).

[14] D. Schlomiuk, Algebraic and geometric aspects of the theory of polynomial vector fields, in "Bifurcations and periodic orbits of vector fields" (Montreal, PQ, 1992), 429-467, Kluwer Acad. Publ., Dordrecht, 1993.

[15] M. Urabe. Potential forces which yield periodic motions of a fixed period, J. Math. Mech. 10 (1961), 569-578.

[16] M. Villarini. Regularity properties of the period function near a centre of a planar vector field, Nonlinear Analysis T.M.A. 19 (1992), 787-803.

[17] E. P. Volokitin and V. V. Ivanov. Isochronicity and commutation of polynomial vector fields, Sibirsk. Mat. Zh. 40 (1999), 30-48. (translation in Siberian Math. J. 40 (1999), 23-38.)

[18] J. Waldvogel. The period in the Lotka-Volterra system is monotonic, J. Math. Anal. Appl. 114 (1986), 178-184. 